\title{
ANALÍTICA DE APRENDIZAJE DE UNA MATERIA OPTATIVA DEL GRADO DE BIOLOGÍA
}

\author{
Becerra-Fernández, Manuel'; González-Siso, María-Isabel² \\ ${ }^{1}$ Universidade da Coruña, Departamento de Bioloxía, Facultade de Ciencias, \\ ORCID: 0000-0003-0913-9749 \\ ${ }^{2}$ Universidade da Coruña, Departamento de Bioloxía, Facultade de Ciencias \\ ORCID: 0000-0001-9538-4841
}

\section{RESUMEN}

El uso de plataformas virtuales de docencia por los estudiantes genera una gran cantidad de huellas y el reto consiste en analizar estos datos para sacar conclusiones del proceso de enseñanzaaprendizaje. En el presente trabajo, nos hemos propuesto analizar los registros que dejan los estudiantes en los espacios virtuales de una materia optativa de cuarto curso del grado de Biología con la finalidad de conocer las actividades que han presentado un mayor número de interacciones y que han despertado por tanto un mayor interés por parte del alumnado.

PALABRAS CLAVE: Analítica de aprendizaje, Moodle, registro de usos.

\section{CITA RECOMENDADA:}

Becerra Fernández, Manuel; González Siso, María Isabel (2021): Analítica de aprendizaje de una materia optativa del grado de Biología. En García Naya, J.A. (ed.) (2021). Contextos universitarios transformadores: a nova normalidade académica. Leccións aprendidas e retos de futuro. V Xornadas de Innovación Docente. Cufie. Universidade da Coruña. A Coruña (pág. 57-66).

DOl capítulo: https://doi.org/10.17979/spudc. 9788497498180.057

DOI libro: https://doi.org/10.17979/spudc.9788497498180 


\section{ABSTRACT}

The use of virtual teaching platforms by students generates a large number of footprints and the challenge is to analyze these data to obtain conclusions about the teaching-learning process. In the present work, we have proposed to analyze the records that students leave in the virtual spaces of an elective subject in the fourth year of the Biology degree in order to know the activities that have presented a greater number of interactions and that have attracted more interest from the students.

KEY WORDS: learning analytics, Moodle, records of uses 


\section{INTRODUCCIÓN}

La incorporación de las TIC (Tecnologías de la Información y la Comunicación) a la docencia y en concreto de herramientas de gestión del aprendizaje como Moodle generan una gran cantidad de información sobre el proceso de enseñanza-aprendizaje. El análisis de estos datos puede propiciar una mejora en el proceso de aprendizaje, en el seguimiento y en la evaluación del estudiante (Ferguson et al., 2016; Sabulsky, 2019).

El estudio de los datos educativos con el propósito de optimizar los procesos de aprendizaje y proporcionar soluciones a cada alumno es lo que se conoce como analítica de aprendizaje 0 Learning Analytics (LA) en inglés (Vela-Pérez et al., 2017).

En la primera conferencia internacional sobre analítica de aprendizaje y conocimiento celebrada en 2011 en Alberta (Canadá) (LAK'11) se definió la analítica de aprendizaje como "la medición, recopilación, análisis y presentación de datos sobre alumnos y sus contextos, con el fin de comprender y optimizar el aprendizaje y los entornos en los que se produce" (https://tekri.athabascau.ca/analytics/).

A partir de los datos de los estudiantes recopilados en un entorno virtual, se hace necesario analizar estos datos con el objetivo de intervenir en el proceso de enseñanza-aprendizaje, adaptando las metodologías a las necesidades del estudiante o del grupo (Villasol, 2019).

En este estudio, se pretende realizar un análisis de los registros que los estudiantes han dejado en la plataforma Moodle dentro de una materia optativa del grado de Biología, Fundamentos Bioquímicos de Biotecnología, que contó con 32 alumnos matriculados.

\section{DESCRIPCIÓN DE LA EXPERIENCIA}

La materia se estructuró en grandes bloques temáticos. Dentro de cada bloque se incluyeron los siguientes recursos:

- archivo PDF con la presentación del tema

- archivo de vídeo con la grabación síncrona del tema 
- material complementario: Recursos bibliográficos, enlaces a páginas web, enlaces a vídeos sobre algunos aspectos del tema y otras actividades complementarias

- Foro de dudas

Las otras actividades complementarias consistieron en juegos de crucigramas, hacer parejas, rellenar huecos, cuestionarios de verdadero/falso. Estas actividades se realizaron con el software para crear ejercicios educativos Hot Potatoes (https://hotpot.uvic.ca/).

Los datos para el análisis se obtuvieron a partir del Moodle de la materia, dentro del apartado de informes que se localiza en la administración del curso. En este trabajo nos hemos centrado en el informe de actividades. Este informe se puede descargar en formato Excel y en él se presentan todas las actividades del curso, el número de visualizaciones totales realizadas, el número de usuarios que han accedido a cada una de ellas y la información del último acceso. Aunque para este trabajo, únicamente nos hemos centrado en el informe de actividades, es de destacar la información que se puede obtener dentro del apartado de registros. En este apartado se puede seleccionar la información individualizada por alumno 0 docente y conocer la actividad asincrónica de los estudiantes: las horas de acceso, actividades consultadas, frecuencias, franjas horarias, tiempos de usos, etc. Esta información también está disponible dentro del apartado de participantes seleccionando al alumno que queremos conocer sus registros, lo que nos permite realizar un seguimiento individualizado y pormenorizado de las actividades del alumno, sus usos y costumbres. Este seguimiento individualizado permite elaborar una estrategia de aprendizaje diferenciada o personalizada.

\section{RESULTADOS}

Del resultado del análisis del informe de actividad obtenido a través de Moodle, se puede destacar que el 89,7\% de los alumnos han accedido al menos una vez a los archivos de los temas en PDF (Figura 1). Sorprende comprobar que haya pocos archivos (guión de prácticas, boletín de problemas) a los que hayan accedido la totalidad del alumnado, probablemente es debido a que se intercambian los archivos entre ellos. 


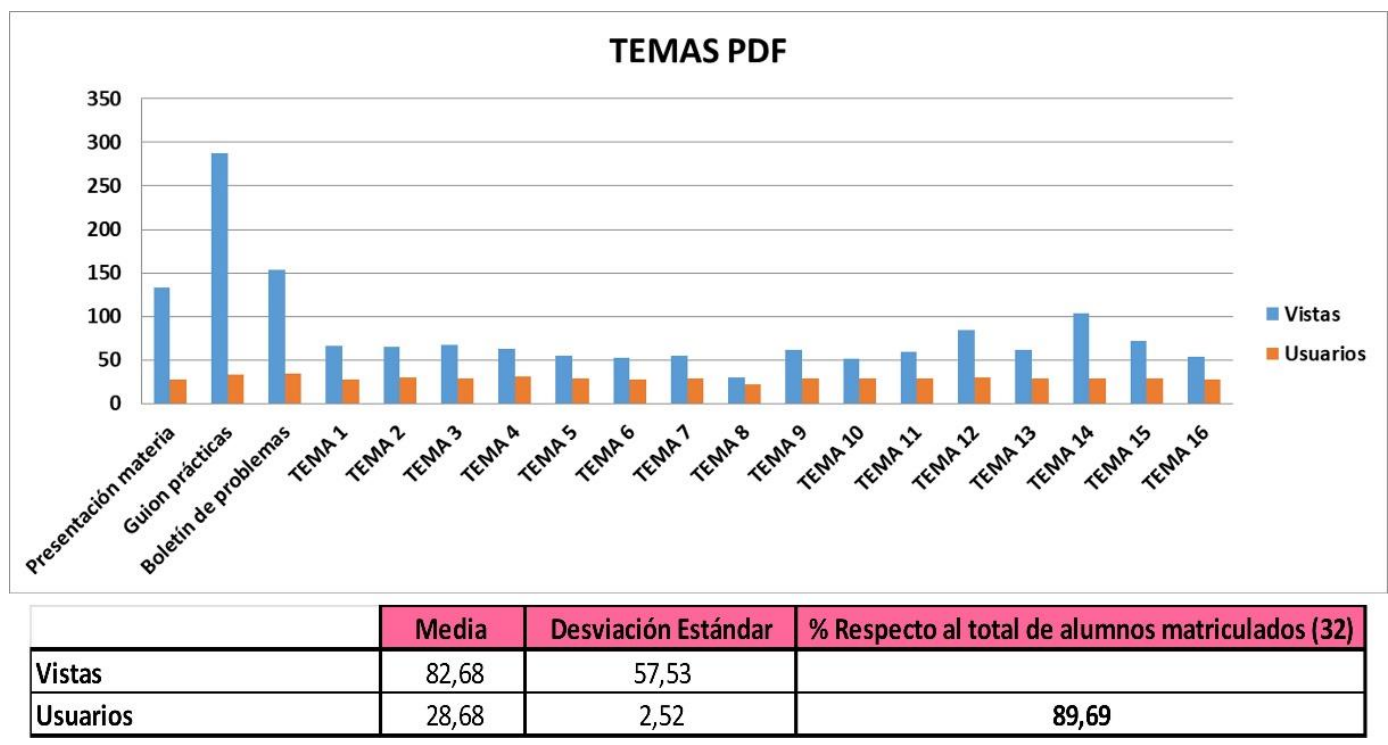

Figura 1: Acceso a los archivos en PDF. En azul se representa el número de visualizaciones, mientras que en naranja está representado el número de usuarios que han accedido a cada uno de los archivos en PDF. En la tabla se describe la media de las visualizaciones y de los usuarios obtenida para los archivos en PDF con su desviación estándar, así como el porcentaje de la media de usuarios con respecto al total de alumnos matriculados.

También es destacable que, a los archivos de los vídeos grabados durante la impartición de las clases sólo han accedido una media de 16 alumnos, lo que representa el 50\% del alumnado (Figura 2). Por lo que estas grabaciones parecen despertar poco interés. Hay que señalar que los datos obtenidos en Moodle nos indican únicamente si se ha accedido al archivo del vídeo en algún momento, pero no muestra si se ha visualizado por completo. Este dato se podría estimar yendo al registro individualizado del alumno y calculando el tiempo de permanencia en cada una de las actividades una vez que ha accedido al curso. 


\section{VIDEOS CLASES SÍNCRONAS Y SEMINARIOS}

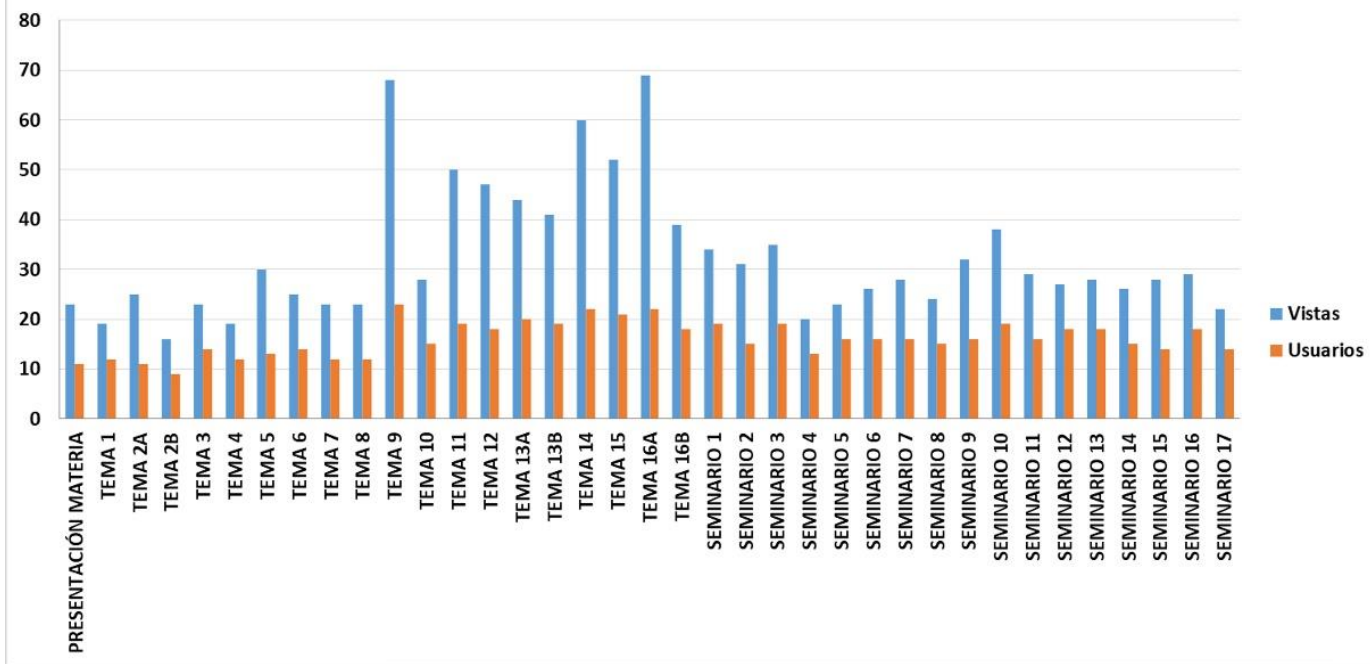

\begin{tabular}{|l|c|c|c|}
\cline { 2 - 4 } \multicolumn{1}{c|}{} & Media & Desviación Estándar & \% Respecto al total de alumnos matriculados (32) \\
\hline Vistas & 32,54 & 13,23 & $\mathbf{5 0 , 1 6}$ \\
\hline Usuarios & 16,05 & 3,45 & \\
\hline
\end{tabular}

Figura 2: Acceso a los vídeos grabados durante la impartición de las clases. En azul se representa el número de visualizaciones, mientras que en naranja está representado el número de usuarios que han accedido a cada uno de los vídeos. En la tabla se describe la media de las visualizaciones y de los usuarios obtenida para los archivos de vídeo con su desviación estándar, así como el porcentaje de la media de usuarios con respecto al total de alumnos matriculados.

Si el acceso a los archivos de las grabaciones de las clases ha sido bajo, el interés despertado por otros recursos como enlaces a páginas web, a artículos científicos o vídeos cortos sobre aspectos concretos de la temática abordada en las clases ha sido muy escaso (Figura 3). Al $11 \%$ de estos recursos no ha accedido ningún alumno, y presentan una media de acceso de 2-3 alumnos lo que supone el 6-9\% de los alumnos matriculados. 

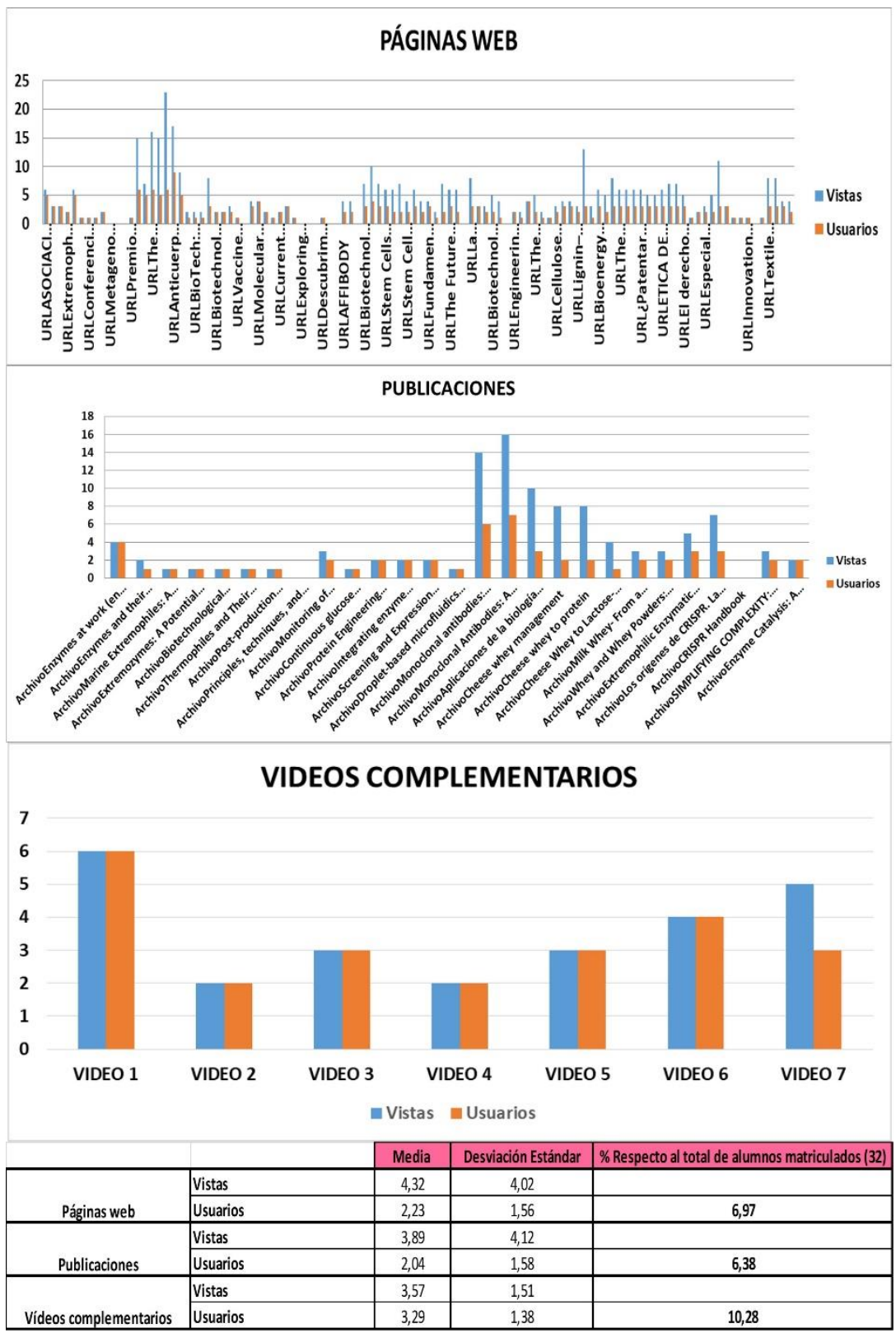

Figura 3: Acceso a enlaces a páginas web, artículos científicos y vídeos cortos complementarios. En azul se representa el número de visualizaciones, mientras que en naranja está representado el número de usuarios que han accedido a cada uno de los recursos. En la tabla se describe la media de las visualizaciones y de los usuarios obtenida para los enlaces a páginas web, artículos científicos y vídeos complementarios con su desviación estándar, así como el porcentaje de la media de usuarios con respecto al total de alumnos matriculados para cada uno de estos recursos. 
Por el contrario, las otras actividades complementarias desarrolladas con el software Hot Potatoes como juegos de crucigramas, hacer parejas, pruebas de verdadero 0 falso...despertaron mayor interés por parte del alumnado, accediendo una media de 23 alumnos lo que supone el $73 \%$ del alumnado (Figura 4).

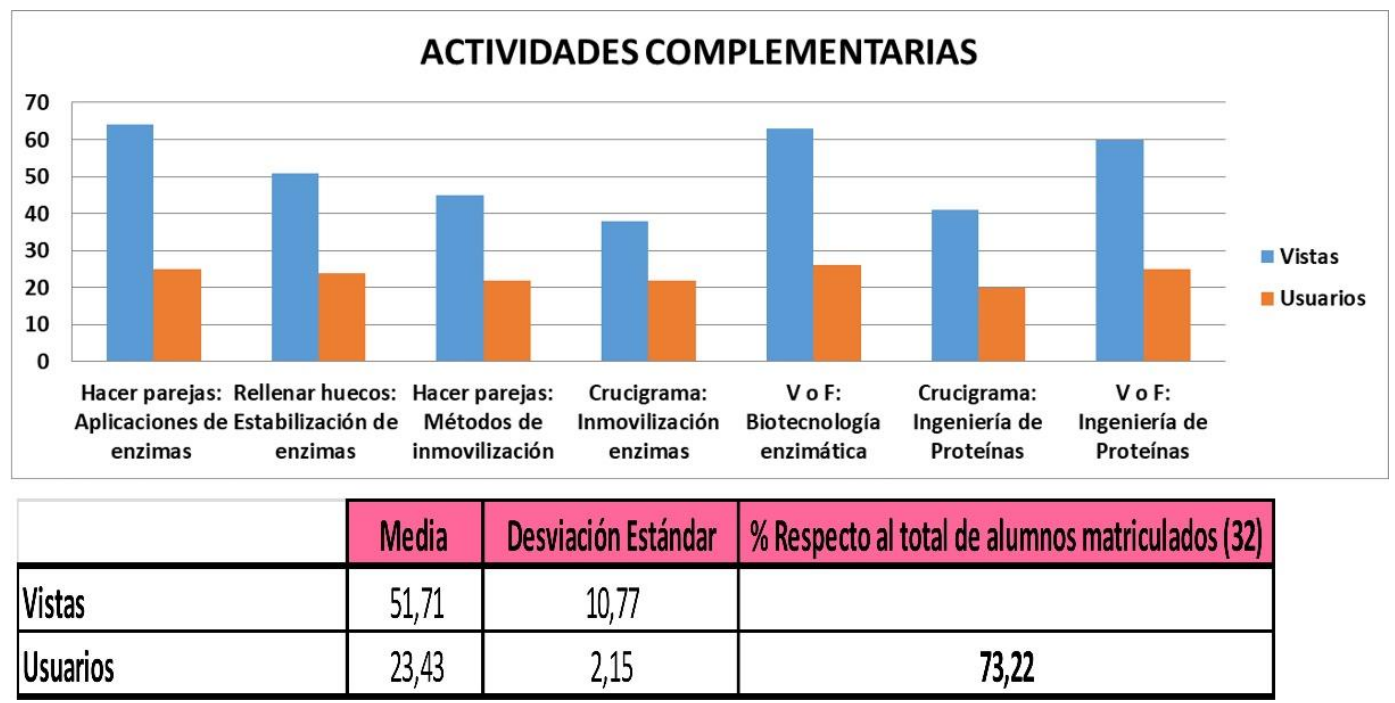

Figura 4: Acceso a actividades complementarias realizadas con el software Hot Potatoes. En azul se representa el número de visualizaciones, mientras que en naranja está representado el número de usuarios que han accedido a cada uno de los recursos. En la tabla se describe la media de las visualizaciones y de los usuarios obtenida para las actividades complementarias con su desviación estándar, así como el porcentaje de la media de usuarios con respecto al total de alumnos matriculados.

Para finalizar, hay que comentar que el acceso a los foros también ha sido muy escaso. No usándose el foro de dudas en ningún momento y únicamente han mostrado interés por el foro de avisos y de novedades, con el $75 \%$ y $78 \%$, respectivamente, de alumnos matriculados que han accedido a ellos (Figura 5). 


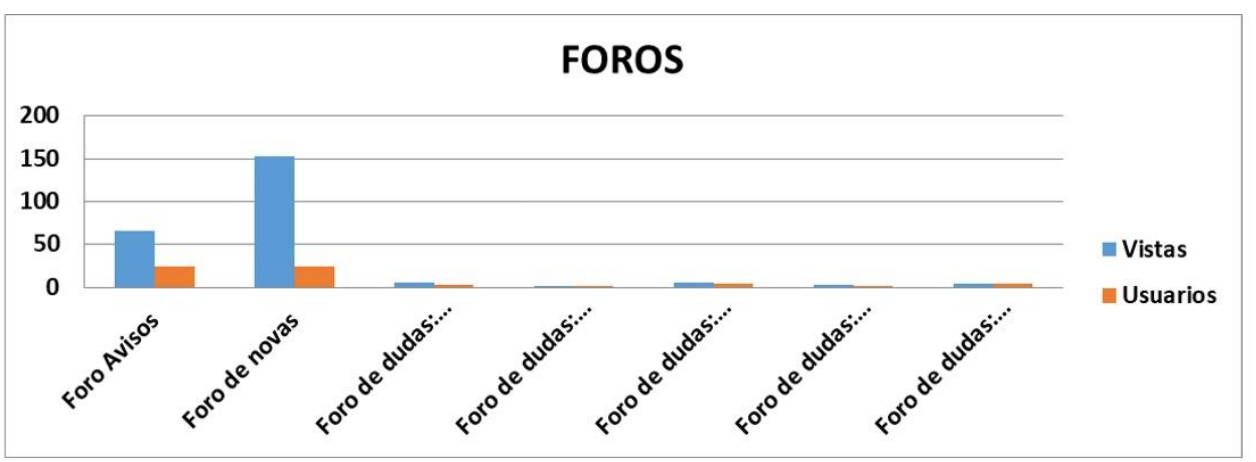

\begin{tabular}{|c|c|c|c|}
\hline & & Valores absolutos & $\%$ Respecto al total de alumnos matriculados (32) \\
\hline \multirow[b]{2}{*}{ Foro de avisos } & Vistas & 66,00 & \\
\hline & Usuarios & 24,00 & 75 \\
\hline \multirow[b]{2}{*}{ Foro de novas } & Vistas & 153,00 & \\
\hline & Usuarios & 25,00 & 78,13 \\
\hline \multirow[b]{2}{*}{ Foro de dudas } & Vistas & 20,00 & \\
\hline & Usuarios & 16,00 & 50 \\
\hline
\end{tabular}

Figura 5: Acceso a los foros. En azul se representa el número de visualizaciones, mientras que en naranja está representado el número de usuarios que han accedido a cada uno de los recursos. En la

tabla se describe la media de las visualizaciones y de los usuarios obtenida para los foros con su desviación estándar, así como el porcentaje de la media de usuarios con respecto al total de alumnos matriculados.

\section{CONCLUSIONES}

El análisis de la impronta virtual que dejan los estudiantes en su actividad asíncrona permite determinar los hábitos de estudio, el uso y preferencias de los recursos educativos lo que posibilita realizar una reflexión y reorientación del proceso de enseñanza-aprendizaje futuro. En este sentido, en este trabajo se ha observado que las presentaciones de los temas en PDF ha sido el recurso más visualizado por los alumnos (el $89,7 \%$ de los mismos han accedido una vez al menos a este tipo de recursos), seguido por actividades complementarias más novedosas realizadas con el software Hot Potatoes (el $73,22 \%$ del alumnado ha accedido a estos recursos). Mientras que a las grabaciones de las clases solamente ha accedido la mitad del alumnado. En lo que respecta a otros recursos como enlaces a páginas web, publicaciones científicas 0 vídeos complementarios el interés ha sido muy bajo ya que únicamente han accedido a ellos entre el $7 \%$ y el $10 \%$ del alumnado. 
Aunque la analítica de aprendizaje es interesante y útil para conocer las preferencias del alumnado y planificar la docencia, presenta la desventaja de ser, en general, poco conocida y usada por parte de los docentes. Uno de los principales retos consiste precisamente en conseguir una mayor utilización de esta. Para ello, se hace necesario disponer de una herramienta sencilla que muestre los datos de una manera que facilite la interpretación de estos por parte del docente.

\section{REFERENCIAS}

Ferguson, R., Brasher, A., Clow, D., Cooper, A., Hillaire, G., Mittelmeier, J., Rienties, B., Ullmann, T. \& Vuorikari, R. (2016). Research evidence on the use of learning analyticsImplications for education policy. R. Vuorikari, J. Castaño Muñoz (Eds.). Joint Research Centre Science for Policy Report; EUR 28294 EN. https://doi.org/10.2791/955210

Sabulsky, G. (2019). Analíticas de Aprendizaje para mejorar el aprendizaje y la comunicación a través de entornos virtuales. Revista Iberoamericana De Educación, 80 (1), 13-30. https://doi.org/10.35362/rie8013340

Vela-Pérez, M., Hernández-Estrada, A., Tirado-Domínguez, G., Martínez-Rodríguez, M. E. \& Peñaloza-Figueroa, J. L. (2017). Learning Analytics to classify students according to their activity in moodle. EDULEARN17 Proceedings, pp. 1166-1172, Barcelona. https://doi.org/10.21125/edulearn.2017.1241

Villasol, M. C. I. (2019). Learning Analytics para una visión tipificada del aprendizaje de los estudiantes. Un estudio de caso. Revista Iberoamericana De Educación, 80 (1), 55-87. https://doi.org/10.35362/rie8013444 